Bull. Austral. Math. Soc.

$20 \mathrm{~K} 40,20 \mathrm{~K} 15,16 \mathrm{~A} 89$

VoL. 38 (1988) [273-291]

\title{
ON THE LATTICE OF RIGHT IDEALS OF THE ENDOMORPHISM RING OF AN ABELIAN GROUP
}

\author{
Theodore G. FATICONI
}

\begin{abstract}
Let $A$ be an abelian group, let $\Lambda=\operatorname{End}(A)$, and assume that $A$ is a flat left $\Lambda$-module. Then $\sigma=\{$ right ideals $I \subset \Lambda \mid I A=A\}$ generates a linear topology on $\Lambda$. We prove that $\operatorname{Hom}\left(A_{1} \cdot\right)$ is an equivalence from the category of those groups $B \subset A^{n}$ satisfying $B=\operatorname{Hom}(A, B) A$, onto the category of $\sigma$-closed submodules of finitely generated free right $\Lambda$-modules. Applications classify the right ideal structure of $A$, and classify torsionfree groups $A$ of finite rank which are (nearly) isomorphic to each $A$-generated subgroup of finite index in $A$.
\end{abstract}

\section{INTRODUCTION}

Modern investigations of direct sum decompositions of a torsion-free abelian group $A$ translate a direct sum decomposition of $A$ into a direct sum decomposition of projective right End $(A)$-modules via the celebrated Theorem of Arnold and Lady [5, Theorem 1.1]. Indeed, before the publication of the Arnold-Lady Theorem, progress in this normally baffling area of abelian groups had been difficult at best. Subsequent investigations have prompted the discussion of interesting properties of $A$ which can be stated in terms of End $(A)$, but not necessarily in terms of direct sum decompositions of $A$. Attempts to apply the Arnold-Lady Theorem to investigations of these new properties do not seem to possess the natural flavour inherent in earlier applications. Specifically, there is a need for a subtler measure of the group structure of $A$ in terms of the right ideal structure of $\operatorname{End}(A)$.

The main result extends the Arnold-Lady Theorem as follows.

Theorem. Let $A$ be an E-flat abelian group, (that is, $A$ is a flat left End $(A)$ module), and let $\mathcal{S}(A)$ denote the category of $A$-generated subgroups of finitely $A$-free groups (that is, $B \in \mathcal{S}(A)$ implies $B=\operatorname{Hom}(A, B) A$ and $B$ embeds as a subgroup of a finite direct sum of copies of $A)$. Let $\mathcal{F}(\operatorname{End}(A))$ denote the category of submodules of finitely generated free right End $(A)$-modules which are closed in the linear topology generated by $\sigma=\{$ right ideals $I$ of $\operatorname{End}(A) \mid I A=A\}$. Then $\operatorname{Hom}(A, \cdot): S(A) \rightarrow$ $\mathcal{F}($ End $(A))$ is a category equivalence with inverse $\cdot \otimes_{\operatorname{End}(A)} A$.

This partial extension of the Arnold-Lady Theorem is enough to ensure intuitive solutions to otherwise difficult problems. For example, call $B \in \mathcal{S}(A)$ finitely $A$ generated if $B=H A$ for some finite subset $H \subseteq \operatorname{Hom}(A, B)$.

Received 7 December, 1987

Copyright Clearance Centre, Inc. Serial-fee code: 0004-9729/88 \$A2.00+0.00. 
Corollary 3.2A. If $A$ is an E-flat group and if $\sigma=\{\operatorname{End}(A)\}$ then End $(A)$ is a Noetherian ring if and only if each $A$-generated subgroup of $B$ is a finitely $A$-generated group.

THEOREM 4.1. End $(A)$ is right semi-hereditary and $A$ is an E-flat group if and only if $\sigma=\{\operatorname{End}(A)\}$ and each finitely $A$-generated subgroup of $A$ is a finitely $A$ projective group.

Corollary 5.6. Let $A$ be a torsion-free group of finite rank. Then End $(A)$ is a classical maximal order if and only if each nonzero $A$-generated subgroup of $A$ generates $A$.

Other results classify those torsion-free groups $A$ of finite rank which are (nearly) isomorphic to each $A$-generated subgroup of finite index in $A$. (See Sections 5 and 6.) Finally, Theorem 1.2 is valid for right modules $A$ over more general associative rings. Because our interests and applications focus on (torsion-free) abelian groups, we forgo the more general setting.

\section{Preliminaries and the main theorem}

Throughout this note; the term group means abelian group. At all times, $n$ denotes a positive integer, $A$ and $B$ denote groups, and $\Lambda=\operatorname{End}(A)$. Consider $A$ as a $\Lambda-\mathbb{Z}$ bimodule, let $\mathcal{H}_{A}(\cdot)=\operatorname{Hom}(A, \cdot)$, let $\mathcal{T}_{A}(\cdot)=\cdot \otimes_{\Lambda} A$, and for sets $H \subseteq \mathcal{H}_{A}\left(A^{n}\right)$ let $H A=\sum_{\lambda \in H} \lambda A$. For torsion-free groups $A$ we let $Q A=A \otimes Q$. The right $\Lambda$-submodule $H$ of $\mathcal{H}_{A}\left(A^{n}\right)$ is called a homset if there is a subgroup $B$ of $A^{n}$ such that $H=\mathcal{H}_{A}(B)$, or equivalently, if $H=\mathcal{H}_{A}(H A)$. The group $B$ is (finitely) $A$-generated if there is a (finite) set of functions $H \in \mathcal{H}_{A}(B)$ such that $B=H A$. Note $B$ is $A$-generated if and only if $B=\mathcal{H}_{A}(B) A$. Finitely $A$-free groups are finite direct sums of copies of $A$, and finitely $A$-projective groups are direct summands of finitely $A$-free groups.

Let $\mathcal{L}\left(A^{n}\right)$ denote the lattice of $A$-generated subgroups of $A^{n}$, and let $\mathcal{S}(A)$ denote the category of A-generated groups which embed in finitely $A$-free groups.

The reader is referred to $[\mathbf{1 9}$, Chapters VI and IX] for a self-contained introduction to linear topologies and torsion theories. However, our use of torsion theory does not extend beyond Section 1 .

Let $\sigma=\sigma(A)$ be the set of right ideals $I$ of $\Lambda$ such that $I A=A$. When $A$ is an $E$-flat group, (that is, when $A$ is a flat left $\Lambda$-module), $\sigma$ is a Gabriel topology on $\Lambda,[19$, Proposition VI.9.3 and p. 156], and thus $\sigma$ defines a hereditary torsion theory and linear topology on right $\Lambda$-modules $M$ as follows. If $\mathcal{T}_{A}(M)=0$ then $M$ is a $\sigma$-torsion module, and the largest $\sigma$-torsion module contained in $M$ is the $\sigma$-torsion submodule of $M$. The $\Lambda$-submodule $N \subset M$ is $\sigma$-dense in $M$ if $M / N$ is a $\sigma$-torsion module. Call $M$ a $\sigma$-closed module if for each $I \in \sigma$ the inclusion $I \subset \Lambda$ induces an 
isomorphism

$$
M \cong \operatorname{Hom}_{\Lambda}(\Lambda, M) \cong \operatorname{Hom}_{\Lambda}(I, M) .
$$

There is a $\sigma$-closed module $M_{\sigma}$ and map $\theta_{M}: M \rightarrow M_{\sigma}$ such that $\theta_{M}(M)$ is $\sigma$-dense in $M_{\sigma}$ and $\operatorname{ker} \theta_{M}$ is the $\sigma$-torsion submodule of $M,[19$, Proposition IX.1.8]. We will call $M_{\sigma}$ the $\sigma$-closure of $M$. If $M$ is a $\sigma$-closed module and if $N \subset M$, then $N_{\sigma} \subset M$ and $N_{\sigma} / N$ is the $\sigma$-torsion submodule of $M / N,[19$, Proposition IX.4.2].

Let $L(M)$ denote the lattice of $\sigma$-closed submodules of $M$, and let $\mathcal{F}(\Lambda)$ denote the category of $\sigma$-closed modules which embed in finitely generated free right $\Lambda$-modules. Observe that our use of the symbol $\mathcal{F}(A)$ is radically different from that in [19].

Lemma 1.1. Assume $A$ is an E-flat group, and let $H \subset$ be right $\Lambda$-submodules of $\mathcal{H}_{A}\left(A^{n}\right)$. Then:

(a) $H$ is $\sigma$-dense in $K$ if and only if $H A=K A$;

(b) $\mathcal{H}_{A}(H A)$ is the $\sigma$-closure of $H$;

(c) $H$ is $\sigma$-closed if and only if $H$ is homset.

Proof: (a) Because $A$ is an E-flat group, $\mathcal{T}_{A}(\cdot)$ is left exact. Thus an inclusion $M \subseteq \mathcal{H}_{A}\left(A^{n}\right)$ of right $\Lambda$-submodules induces the embedding

$$
\mathcal{T}_{A}(M) \rightarrow \mathcal{T}_{A}\left(\mathcal{H}_{A}\left(A^{N}\right)\right) \cong \mathcal{H}_{A}\left(A^{n}\right) A=A^{n}
$$

where $f \otimes a \in \mathcal{T}_{A}(M)$ is mapped to $f(a) \in A^{n}$. Hence $\mathcal{T}_{A}(M) \cong M A$. Therefore applying $\mathcal{T}_{A}(\cdot)$ to the short exact sequence $0 \rightarrow H \stackrel{\lambda}{\rightarrow} K \rightarrow K / H \rightarrow 0$ shows that $H A=K A$ if and only if $\mathcal{T}_{A}(\lambda)$ is an isomorphism if and only if $\mathcal{T}_{A}(K / H)=0$ if and only if $H$ is $\sigma$-dense in $K,[19$, p. 156].

(b) Let $I \in \sigma$. By part (a) $\mathcal{T}_{A}(I) \cong \mathcal{T}_{A}(\Lambda)$. Then there are natural (adjoint) isomorphisms

$$
\operatorname{Hom}_{\Lambda}\left(I, \mathcal{H}_{A}\left(A^{n}\right)\right) \cong \operatorname{Hom}\left(\mathcal{T}_{A}(I), A^{n}\right) \cong \operatorname{Hom}\left(\mathcal{T}_{A}(\Lambda), A^{n}\right) \cong \operatorname{Hom}_{\Lambda}\left(\Lambda, \mathcal{H}_{A}\left(A^{n}\right)\right)
$$

for each positive integer $n$. Thus $\mathcal{H}_{A}\left(A^{n}\right)$ is a $\sigma$-closed module, and so $H \subset H_{\sigma} \subset$ $\mathcal{H}_{A}\left(A^{n}\right)$. By part (a) $H A=H_{\sigma} A$, so that $H_{\sigma} \subset \mathcal{H}_{A}(H A)$. Inasmuch as $\mathcal{H}_{A}(H A) A=$ $H A$, part (a) shows that $H$ is $\sigma$-dense in $\mathcal{H}_{A}(H A)$. Because $H_{\sigma} / H$ is the $\sigma$-torsion submodule of $\mathcal{H}_{A}\left(A^{n}\right) / H, \mathcal{H}_{A}(H A)=H_{\sigma}$.

(c) By part (b) $H=\mathcal{H}_{A}(H A)$ if and only if $H$ is $\sigma$-closed.

From Lemma 1.1c it is clear that $\Lambda$ is a $\sigma$-closed module, and hence each finitely generated projective right $\Lambda$-module is $\sigma$-closed. However, for some values of $\sigma$ and $A, \sigma$-closed modules need not be projective as the following shows. Let $\Lambda$ be a reduced torsion-free finite rank ring which is not right hereditary. Since $\Lambda$ is not right hereditary, there is a nonprojective right ideal $I$ of $\Lambda$. By [8, Corollary 3.9] there is an E-flat group $A$ for which $\Lambda=\operatorname{End}(A)$ and $\sigma=\{\Lambda\}$. In this case each right ideal of $\Lambda$ is $\sigma$-closed, so $I$ is $\sigma$-closed. 
TheOREM 1.2. Let $A$ be an E-flat group. Then:

(a) $\mathcal{H}_{A}(\cdot): \mathcal{S}(A) \rightarrow \mathcal{F}(\Lambda)$ and $\mathcal{T}_{a}(\cdot): \mathcal{F}(\Lambda) \rightarrow \mathcal{S}(A)$ are inverse category equivalences;

(b) For each positive integer $n, \mathcal{H}(\cdot): \mathcal{L}\left(A^{n}\right) \rightarrow \mathcal{L}\left(\Lambda^{n}\right)$ is a lattice isomorphism.

Proof: (a) Let $B \in \mathcal{S}(A)$ and let $H \in \mathcal{F}(\Lambda)$. By Lemma 1.1c, $\mathcal{H}_{A}(B) \in \mathcal{F}(\Lambda)$, so that $\mathcal{H}_{A}(\cdot): \mathcal{S}(A) \rightarrow \mathcal{F}(\Lambda)$ is well-defined. Given an embedding $\theta: H \rightarrow \Lambda^{n} \cong$ $\mathcal{H}_{A}\left(A^{n}\right), \theta(H) \subset \mathcal{H}_{A}\left(A^{n}\right)$ is a $\sigma$-closed module. Then by Lemma 1.1c $\theta(H)=\mathcal{H}_{A}(C)$ for some $C \in \mathcal{L}\left(A^{n}\right)$. Because $\mathcal{T}_{A}(\cdot)$ is left exact

$$
\mathcal{T}_{A}(\theta): \mathcal{T}_{A}(H) \rightarrow \mathcal{T}\left(\mathcal{H}_{A}\left(A^{n}\right)\right) \cong A^{n}
$$

is an embedding with image $\mathcal{H}_{A}(C) A=C$. Thus $\mathcal{T}_{A}(H) \cong C \in \mathcal{L}\left(A^{n}\right)$, and so $\mathcal{T}_{A}(\cdot): \mathcal{F}(\Lambda) \rightarrow \mathcal{S}(A)$ is well-defined.

Now, it is well-known that the map $\mathcal{T}_{A}\left(\mathcal{H}_{A}(B)\right) \rightarrow B$ sending $f \otimes a \in \mathcal{T}_{A}\left(\mathcal{H}_{A}(B)\right)$ to $f(a) \in B$ is a natural homomorphism. Since $B$ is $A$-generated and since $\mathcal{T}_{A}(\cdot)$ is left exact, $\mathcal{T}_{A}\left(\mathcal{H}_{A}(B)\right) \cong \mathcal{H}_{A}(B) A=B$ as in the proof of Lemma 1.1a. Thus $\mathcal{T}_{A}\left(\mathcal{H}_{A}(\cdot)\right)$ is naturally equivalent to the identity functor on $\mathcal{S}(A)$.

As above, an embedding $H \rightarrow \mathcal{H}_{A}\left(A^{n}\right)$ has image $\mathcal{H}_{A}(C)$ for some $C \in \mathcal{L}\left(A^{n}\right)$, and $\mathcal{T}_{A}\left(\mathcal{H}_{A}(C)\right) \cong C$ naturally. Because $H \cong \mathcal{H}_{A}(C)$ there are natural isomorphisms $\mathcal{H}_{A}\left(\mathcal{T}_{A}(H)\right) \cong \mathcal{H}_{A}\left(\mathcal{T}_{A}\left(\mathcal{H}_{A}(C)\right)\right) \cong \mathcal{H}_{A}(C) \cong H$. Thus $\mathcal{H}_{A}\left(\mathcal{T}_{A}(\cdot)\right)$ is naturally equivelent to the indentity functor on $\mathcal{F}(\Lambda)$. This proves that $\mathcal{H}_{A}(\cdot): \mathcal{S}(A) \rightarrow \mathcal{F}(\Lambda)$ is a category equivelence with inverse $\mathcal{T}_{A}(\cdot): \mathcal{F}(\Lambda) \rightarrow \mathcal{S}(A)$.

(b) We identify $\Lambda^{n} \cong \mathcal{H}_{A}\left(A^{n}\right)$. Define $h: \mathcal{L}\left(A^{n}\right) \rightarrow \mathcal{L}\left(\mathcal{H}_{A}\left(A^{n}\right)\right)$ by $h(B)=$ $\mathcal{H}_{A}(B)$, and $h^{\prime}: \mathcal{L}\left(\mathcal{H}_{A}\left(A^{n}\right)\right) \rightarrow \mathcal{L}\left(A^{n}\right)$ by $h^{\prime}(H)=H A$. Then given $H \in \mathcal{L}\left(\mathcal{H}_{A}\left(A^{n}\right)\right)$ Lemma 1.1c implies $h h^{\prime}(H)=\mathcal{H}_{A}(H A)=H$, while each $B \in \mathcal{L}\left(A^{n}\right)$ satisfies $h^{\prime} h(B)=$ $\mathcal{H}_{A}(B) A=B$. Thus $h$ is a bijection, and since $h$ preserves inclusion, $h$ is a lattice isomorphism.

Remark. By the comments preceeding it, Theorem 1.2 is a proper extension (for E-flat groups) of the Arnold-Lady Theorem [5, Theorem 1:1].

The hypothesis that $A$ must be an E-flat group is not as restrictive as it may first appear to be. For example, by [8, Proposition 2.2] each strongly indecomposable torsion-free group of rank two is an E-flat group, and by [8, Corollary 3.9] each reduced torsion-free finite rank ring $\Lambda$ is the endomorphism ring of an E-flat group. For torsion groups $A$ there is the following example due to Richman and Walker [18]. Our proof is somewhat simpler.

Example 1.3. Let $A$ be a torsion group.

(a) Let $A_{p}$ be the $p$-torsion part of $A$. Then $A$ is an E-flat group if and 
only if $A_{p}$ is an E-flat group for each prime $p \in \mathbf{Z}$.

(b) Let $A$ be a $p$-group. Then $A$ is not an E-flat group if and only if $A=B \oplus D$ where $D \neq 0$ is a divisible group and $B$ is a bounded group.

(c) If $A$ is an E-flat group then each subgroup of $A$ is $A$-generated.

Proof: (a) Since $A=\oplus_{p} A_{p}$ as left $\Lambda$-modules, and since tensor products commute with direct sums, the result is clear.

(b) Both implications are proved using the Proposition of [7]: $A$ is an E-flat group if and only if $A$ generates the kernel of each map $A^{n} \rightarrow A, n>0$.

Assume $A=B \oplus D$ where $D \neq 0$ is a divisible group and $B$ is a bounded group. If $B p^{k}=0$ then $D\left[p^{k+1}\right]$ (the kernel of the map sending $x \in D$ to $x p^{k+1}$ ) is not generated by $B$ since $D\left[p^{k+1}\right]$ has elements of order $p^{k+1}$. By [7] $A$ is not an E-flat group.

Conversely, we prove the contrapositive. Assume $A=B \oplus D$ where $D$ is the divisible part of $A$ and either $A$ is bounded or $B$ is unbounded. Let $C=C_{1} \oplus C_{2} \oplus \ldots$ be a basic subgroup of $A$ where $C_{k}$ is a direct sum of copies of $\mathbf{Z} / \mathbf{Z} p^{k}$ of $C_{k}=0$. Then

$$
A=C_{1} \oplus \ldots \oplus C_{k} \oplus\left(C_{k+1}+A p^{k+1}\right)
$$

for each $k$. If $A$ is bounded then, for some $k, A=C_{1} \oplus \ldots \oplus C_{k}$. If $B$ is unbounded then $C_{k} \neq 0$ for infinitely many $k$. In either case, for $x \in A^{n}$ (of finite order) there is an integer $k$ and a surjection $C_{k} \rightarrow x \mathbb{Z}$ which lifts to a surjection $A \rightarrow x \mathbb{Z}$. That is $A$ generates each subgroup of $A^{n}$, and therefore $A$ is an E-flat group.

(c) See the proof of part (b).

Corollary 1.4. Let $A$ be a torsion, E-flat group. Then $\mathcal{L}(\Lambda)$ is lattice isomorphic to the lattice of subgroups of $A$.

Proof: Use Theorem 1.2b and Example 1.3.

There is a torsion-free group $A$ possessing a pure subgroup $B$ such that $A / B$ embeds in $A$ but $B / \operatorname{Hom}(A, B) A$ is finite and nonzero, [8, Example 4.5].

\section{Finitely faithful groups}

We call $A$ finitely faithful if $\sigma=\{\Lambda\}$. Our use of this term is different from that in [2] and [3]. However, Lemma 3.1a will show that a torsion-free group $A$ of finite rank is finitely faithful in our sense if and only if it is finitely faithful in the sense of [3] if and only if it is faithful in the sense of [2]. Finitely faithful groups were introduced in [5] to classify the splitting of short exact sequences $0 \rightarrow B \rightarrow G \rightarrow A \rightarrow 0$ in which $B+\mathcal{H}_{A}(G) A=G$. See also $[\mathbf{1}, \mathbf{8}, \boldsymbol{\theta}]$. Our applications of Theorem 1.2 and Corollary $2.2 b$ cast the finitely faithful property in a new light.

An extension of [3, Proposition 4.2] classifies finitely faithful E-flat groups. 
Proposition 2.1. Assume $A$ is an E-flat group. Then the following are equivalent:

(a) A is a finitely faithful group;

(b) Each maximal right ideal of $\Lambda$ is a homset;

(c) Each right ideal of $\Lambda$ is a homset;

(d) Given a positive integer $n$, each right $\Lambda$-submodule of $\mathcal{H}_{A}\left(A^{n}\right)$ is a homset.

Proof: (a) $\Leftrightarrow$ (b) is [3, Proposition 4.2]. Assuming (a), note that each right $\Lambda$-module is $\sigma$-closed. Thus, Theorem $1.2 \mathrm{~b}$ shows that given a right $\Lambda$-submodule $H \subseteq \mathcal{H}_{A}\left(A^{n}\right)$ there is a $B \in \mathcal{L}\left(A^{n}\right)$ such that $H=\mathcal{H}_{A}(B)$. Hence (a) $\Rightarrow$ (d). The implications (d) $\Rightarrow$ (c) $\Rightarrow$ (b) are clear.

A functor that has been useful in studying quasi-direct summands of torsion-free abelian groups $A$ of finite rank is $\mathrm{QH}_{A}(\cdot)=\mathrm{Q} \otimes \operatorname{Hom}(A, \cdot)$, taking the category of quasi-summands of finitely $A$-projective groups to the category of finitely generated projective right $Q \Lambda$-modules. (See [2, Chapter 5] or [16]. [10] contains a noncommutative version of this result.) Let $P \mathcal{L}\left(A^{n}\right)$ be the sublattice of $L(A)$ whose elements are pure subgroups of $A^{n}$, and let $P \mathcal{L}\left(\Lambda^{n}\right)$ be the sublattice of $\mathcal{L}\left(\Lambda^{n}\right)$ whose elements are pure subgroups of $\Lambda^{n}$. Call the elements of $P \mathcal{L}\left(\Lambda^{n}\right)$ pure right submodules of $\Lambda^{n}$.

Corollary 2.2. Let $A$ be a torsion-free E-flat group.

(a) For each integer $n, \mathcal{H}_{A}(\cdot): P \mathcal{L}\left(A^{n}\right) \rightarrow P \mathcal{L}\left(\Lambda^{n}\right)$ is a lattice isomorphism;

(b) $A$ is a finitely faithful group if and only if for each $n, \mathcal{H}_{A}(\cdot)$ is a lattice isomorphism from $\mathcal{L}\left(A^{n}\right)$ onto the lattice of right $\Lambda$-submodules of $\Lambda^{n}$;

(c) If $A$ is a finitely faithful group, then $Q \mathcal{H}_{A}(\cdot)$ is a lattice isomorphism from $P \mathcal{L}\left(A^{n}\right)$ onto the lattice of right $Q \Lambda$-submodules of $Q \Lambda^{n}$.

Proof: (a) It is well-known that if $B$ is a pure subgroup of the torsion-free group $A^{n}$, then $\mathcal{H}_{A}(B)$ is a pure subgroup of $\mathcal{H}_{A}\left(A^{n}\right)$. Thus $\mathcal{H}_{A}(\cdot): P \mathcal{L}\left(A^{n}\right) \rightarrow P \mathcal{L}\left(\Lambda^{n}\right)$ is well-defined. If $H=\mathcal{H}_{A}(B)$ is a pure submodule of $\mathcal{H}_{A}\left(A^{n}\right)=\Lambda^{n}$ then an application of the left exact functor $\mathcal{T}_{A}(\cdot)$ to the short exact sequence $0 \rightarrow \Lambda^{n} / H \rightarrow \mathbb{Q} \Lambda^{n} / \mathrm{Q} H$ yields the embedding $A^{n} / B=A^{n} / H A \rightarrow Q A^{n} / \mathrm{Q} H A$, (Theorem 1.2b). Thus, $A^{n} / B$ is torsion-free, and hence $B$ is pure in $A^{n}$. That is, $\mathcal{H}_{A}(\cdot)$ takes $P \mathcal{L}\left(A^{n}\right)$ onto $P \mathcal{L}\left(\Lambda^{n}\right)$. Now apply Theorem $1.2 b$.

(b) Follows from Proposition 2.1, while (c) follows from part (a) and the fact that $H \rightarrow \mathrm{Q} \otimes H$ is an isomorphism from the lattice of pure right submodules of $\Lambda^{n}$ onto the lattice of right $Q \Lambda$-submodules of $Q \Lambda^{n}$.

The following extension of Proposition 2.1 will prove useful. 
Lemma 2.3. Let $A$ be a group. Then the following are equivalent:

(a) $I A \neq A$ for each finitely generated proper right ideal $I$ of $A$;

(b) Given a positive integer $n, H A \neq A^{n}$ for each finitely generated proper right $\Lambda$-submodule $H$ of $\mathcal{H}_{A}\left(A^{n}\right)$.

ProOF: (b) $\Rightarrow(\mathrm{a})$ is clear. To prove (a) $\Rightarrow(\mathrm{b})$ we use induction on $n$. If $n=1$, then $H A \neq A$ by part (a). Assume part (b) is true for each integer $k<n$ and let $H$ be a finitely generated proper $\Lambda$-submodule of $\mathcal{H}_{A}\left(A^{n}\right)$. Fix a decomposition $A^{n}=A_{1} \oplus \ldots \oplus A_{n}$ where $A_{i} \cong A$ for $1 \leqslant i \leqslant n$. We consider two cases.

Case 1. $H+\mathcal{H}_{A}\left(A_{1}\right)=\mathcal{H}_{A}\left(A^{n}\right)$. Then $H=K_{1} \oplus H_{1}$ where $K_{1}=\mathcal{H}_{A}\left(A_{1}\right) \cap H$ and $H_{1} \cong H / K_{1} \cong \mathcal{H}_{A}\left(A^{n}\right) / \mathcal{H}_{A}\left(A_{1}\right) \cong \Lambda^{n-1}$. Thus $\mathcal{H}_{A}\left(A^{n}\right)=H+\mathcal{H}_{A}\left(A_{1}\right)=$ $H_{1} \oplus \mathcal{H}_{A}\left(A_{1}\right)$ as right $\Lambda$-modules. Observe that $K_{1}$ is finitely generated. Now because $H \neq \mathcal{H}_{A}\left(A^{n}\right), K_{1} \neq \mathcal{H}_{A}\left(A_{1}\right)$, so by part (a) $K_{1} A \neq A_{1}$. Thus

$$
A^{n}=H_{1} A \oplus A_{1} \neq H_{1} A \oplus K_{1} A=H A \text {. }
$$

Case 2. $H+\mathcal{H}_{A}\left(A_{1}\right) \neq \mathcal{H}_{A}\left(A^{n}\right)$. Then identify $\bar{H}=\left(H+\mathcal{H}_{A}\left(A_{1}\right) \mathcal{H}_{A}\left(A_{1}\right)\right)$ with a finitely generated proper $\Lambda$-submodule of $\mathcal{H}_{A}\left(A^{n-1}\right) \cong \mathcal{H}_{A}\left(A^{n}\right) / \mathcal{H}_{A}\left(A_{1}\right)$ in the natural way. The induction hypothesis states that $\bar{H} A \neq A^{n-1}$, and hence $H A \neq A^{n}$. This completes the proof.

The following extends [2, Theorem 5.6].

Proposition 2.4. Let $A$ be a group. Then the following are equivalent:

(a) $I A \neq A$ for each finitely generated proper right ideal $I$ of $\Lambda$;

(b) if $\mathcal{E}: 0 \rightarrow B \rightarrow G \stackrel{\pi}{\rightarrow} A \rightarrow 0$ is a short exact sequence, if $\mathcal{H}_{A}(G) A$ is a finitely $A$-generated group, and if $G=B+\mathcal{H}_{A}(G) A$ then $\mathcal{E}$ is split exact.

Proof: Assume part (a). Given a sequence $\mathcal{E}$ as in part (b), there is a finitely generated right $\Lambda$-submodule $H \subset \mathcal{H}_{A}(G)$ such that $\mathcal{H}_{A}(G) A=H A$. Then $\pi H$ is a finitely generated right ideal of $\Lambda$ such that $\pi H A=\pi(B+H A)=A$. By part (a) $\pi H=\Lambda$, so there exists a map $\lambda \in H$ such that $\pi \lambda=1_{A}$. That is, $\mathcal{E}$ is split exact.

Assume part (a) is false, and let $I=\sum_{i=1}^{n} \pi_{i} \Lambda$ be a finitely proper generated right ideal of $\Lambda$ such that $I A=A$. Define a map $\pi: A^{n} \rightarrow A$ by $\pi\left(a_{1}, \ldots, a_{n}\right)=\sum_{i=1}^{n} \pi_{i}\left(a_{i}\right)$. Then $\pi$ is onto, since $\sum_{i} \pi_{i} A=I A=A$. Now given a map $\lambda: A \rightarrow A^{n}$, write $\lambda=\left(\lambda_{1}, \ldots, \lambda_{n}\right)$ where $\lambda_{i}$ is the projection of $\lambda$ into the $i^{\text {th }}$ copy of $A$ in $A^{n}$. Then $\pi \lambda=\sum_{i} \pi_{i} \lambda_{i} \neq 1_{A}$ since $I \neq \Lambda$. Thus $\pi$ does not split, and the negation of (b) follows. 


\section{SPecial lattices of Right ideals}

In this Section, we classify certain properties of $\Lambda$ in terms of the group structure of $A$.

The ring $\Lambda$ is local if $\Lambda$ contains a unique maximal right ideal, and $\Lambda$ is $s u b$ commutative if for each $x, y \in \Lambda$ there exists a $z \in \Lambda$ such that $y x=x z$. Call $\Lambda$ a right valuation ring if for each $x, y \in \Lambda$ either $x \Lambda \subseteq y \Lambda$ or $y \Lambda \subseteq x \Lambda$. A right valuation domain is a right valuation ring without nonzero zero divisors. Note that $\Lambda$ is subcommutative if and only if each right ideal of $\Lambda$ is a left ideal, and $\Lambda$ is a right valuation ring if and only if the right ideals of $\Lambda$ form a chain. Subcommutative rings are discussed in [15] and the figure in the classification of strongly homogeneous groups offered in [3, Section 3].

Lemma 3.1. Let $A$ be a reduced torsion-free group of finite rank and let $I$ be a maximal right ideal of $\Lambda$. Then:

(a) $\Lambda / I$ is finite;

(b) Assume there exists up to isomorphism exactly one simple right $\Lambda / \Lambda p$ module for each prime $p \in \mathbb{Z}$. Then $A$ is a finitely faithful group;

(c) If $\Lambda$ is a subcommutative ring, then $A$ is a finitely faithful group.

Proof: (a) Let $N$ be the nil radical of $\Lambda$. Since $\Lambda / N$ is a quasi-summand of $\Lambda,[2$, Theorem 14.2], and since $A$ is reduced, $\Lambda / N$ is reduced. Since $I$ is maximal, $N \subset I$, so that $\Lambda / I \cong(\Lambda / N) /(I / N)$. Thus there is no loss of generality in assuming that $\Lambda$ is a reduced semi-prime ring.

Now, because $\Lambda / I$ is a simple right $\Lambda$-module, $\operatorname{End}_{\Lambda}(\Lambda / I)$ is a division ring, and so $\Lambda / I$ is a finite dimensional vector space over $Q$ or $\mathbb{Z} / \mathbb{Z} p$ for some prime $p \in \mathbb{Z}$. But pure right ideals of the semi-prime ring $\Lambda$ are quasi-summands and $\Lambda$ is a reduced ring. Thus $\Lambda / I$ is finite.

(b) Recall [8, Lemma 1.7d]: if $I A=A$ then $\{\lambda \in \mathbb{Q} \Lambda: \mid \lambda I \subseteq \Lambda\}=\Lambda$. By part (a) $\Lambda / I$ is a finite simple right $\Lambda$-module. Let $p \in \mathbb{Z}$ be the prime such that $p \Lambda \subset I$, and let $S$ be a simple $\Lambda / \Lambda p$-submodule of the finite $\Lambda / \Lambda p$-module $\Lambda p^{-1} / \Lambda$. By hypothesis there exists up to isomorphism exactly one simple right $\Lambda / \Lambda p$-module, so $S \cong \Lambda / I$. But $I+1$ corresponds to an element $0 \neq \Lambda+\lambda \in \Lambda p^{-1} / \Lambda$ such that $\lambda I \subset \Lambda$. By $[8$, Lemma $1.7 \mathrm{~d}], I A \neq A$, so that $A$ is a finitely faithful group.

(c) By part (a), $\Lambda p \subset I$ for some prime $p \in \mathbb{Z}$. Since $\Lambda / \Lambda p$ is a finite (Artinian) ring, the Jacobson radical $J / \Lambda p$ of $\Lambda / \Lambda p$ is nilpotent. Now $J \subset I$, and because $\Lambda / J$ is a semi-simple Artinian ring, $I / J=\bar{e}(\Lambda / J)$ for some idlempotent $1 \neq \bar{e} \in \Lambda / J$. Because $J / \Lambda p$ is nilpotent, $\bar{e}=(J / \Lambda p)+e$ for some idempotent $1 \neq e \in \Lambda / \Lambda p$, and because $\Lambda / \Lambda p$ is subcommutative $e(\Lambda / \Lambda p)$ is an ideal of $\Lambda / \Lambda p$. Observe that $I / \Lambda p=e(\Lambda / \Lambda p)+J / \Lambda p \neq \Lambda / \Lambda p$. 
We have

$$
I A / A p=[I / \Lambda p](A / A p)=[e(\Lambda / \Lambda p)+J / \Lambda p](A / A p)=e(A / A p)+[J / \Lambda p](A / A p)
$$

by our choice of $e$. Because $A / A p$ is finite and because $I / \Lambda p$ and $e(\Lambda / \Lambda p)$ are ideals of $\Lambda / \Lambda p$, we may apply Nakayana's Lemma to show that $I A / A p=e(A / A p)$. But then $1-e \neq 0$ annihilates $I A / A p \subset A / A p$. Since $A / A p$ is a faithful left $\Lambda / \Lambda p$-module, $I A / A p \neq A / A p$. Therefore $I A \neq A$ and $A$ is a finitely faithful group.

Remark. Lemma 3.1 extends [5, Corollary 2.3] which states that if $A$ is a torsion-free group of finite rank and if $\Lambda$ is either a commutative or right hereditary ring then $A$ is a finitely faithful group.

With these technical results out of our way, we can classify some common types of endomorphism ring.

Corollary 3.2. Let $A$ be an E-flat group.

(a) Assume $A$ is finitely faithful. Then $\Lambda$ is a right Noetherian ring if and only if each $B \in \mathcal{L}(A)$ is a finitely $A$-generated group.

(b) Assume $A$ is torsion-free of finite rank. Then $\Lambda$ is a local ring if and only if $A$ is a finitely faithful group and there exists a unique maximal $B \in \mathcal{L}(A)$ such that $B \neq A$.

Proof: (a) The classic argument shows that $\mathcal{L}(A)$ is a Noetherian lattice if and only if each $B \in \mathcal{L}(A)$ is a finitely $A$-generated group. Thus (a) follows from Corollary 2.2b. (b) follows from Corollary $2.2 b$ and Lemma $3.1 \mathrm{~b}$.

Corollary 3.3. Let $A$ be an E-flat group.

(a) Assume $A$ is a finitely faithful group. Then $\Lambda$ is a subcommutative ring if and only if each $B \in \mathcal{L}(A)$ is fully invariant in $A$.

(b) Assume $A$ is torsion-free of finite rank. Then $\Lambda$ is a subcommutative ring if and only if $A$ is a finitely faithful group such that each $B \in \mathcal{L}(A)$ is fully invariant in $A$.

PROOF: As each right ideal in a subcommutative ring is an ideal, the Corollary follows from Corollary 2.2b and Lemma 3.1c.

Remark. Corollaries 3.2 and 3.3 seem to be new. They are typical of the intuitive solutions produced by Theorem 1.2 when applied to the problem of classifying properties of endomorphism rings. (See [11, Problem 84].)

In contrast, the next result makes little use of Theorem 1.2. 


\section{Proposition 3.4.}

(a) Assume $A$ is a finitely faithful E-flat group. Then $\Lambda$ is a valuation ring if and only if $\mathcal{L}(A)$ is a chain.

(b) Assume $A$ is a torsion-free group of finite rank. Then $\Lambda$ is a right valuation ring if and only if $\mathcal{L}(A)$ is a chain. In this case, $\Lambda$ is a local subcommutative principal right ideal domain, and $A$ is a finitely faithful E-flat group.

Proof: (a) $\Lambda$ is a right valuation ring if and only if the right ideals of $\Lambda$ form a chain. Thus part (a) follows from Corollary $2.2 \mathrm{~b}$.

(b) $(\Rightarrow)$ If $\Lambda$ is a right valuation ring then $\mathcal{L}(A)=\{I A \mid$ right ideals $I \subseteq \Lambda\}$ is a chain.

$(\Leftarrow)$ Assume that $A$ is a torsion-free group of finite rank in which $\mathcal{L}(A)$ is a chain. Observe that $A$ is strongly indecomposable since in a quasi-decomposition $A \doteq$ $B \oplus C$ with $B \neq 0$, the chain condition in $\mathcal{L}(A)$ and the equation $\operatorname{Hom}(A, B) A \cap$ $\operatorname{Hom}(A, C) A \subseteq B \cap C^{\prime}=0$ imply $C=0$. Thus $Q \Lambda$ is a local ring, [2, Corollary 7.8 ]. We claim that $\mathrm{Q} \Lambda$ is a division ring. Let $N$ be the nilradical of $\Lambda$. Then $\mathrm{QA} \neq$ $(\mathbb{Q} N) \mathbb{Q} A=\mathbb{Q} N A$ by Nakayama's Lemma. Because $\mathcal{L}(A)$ is a chain, $\mathbb{Q} N A \cap A \subset A p$ for all primes $p \in \mathbb{Z}$. But $\mathbb{Q} N A \cap A$ is a pure subgroup of $A$, so $\mathbb{Q} N A \cap A$ is divisible. Since $A$ is reduced $\mathbb{Q} N A \cap A=0$, and since $A$ is a torsion-free group, $N=0$. Thus $\mathrm{Q} \Lambda$ is a division ring as claimed. Let $\lambda, \gamma \in \Lambda$. Since $\mathcal{L}(A)$ is a chain we may assume, without loss of generality that $\lambda A \subset \gamma A$. Now because $Q \Lambda$ is a division ring $\gamma^{-1} \in \mathbb{Q} \Lambda$, and thus $\gamma^{-1} \mathbb{Q} A \subseteq \mathbb{Q} A$. Hence $\gamma^{-1} \lambda(A) \subseteq A$ which implies that $\gamma^{1} \lambda \Lambda \subseteq \Lambda$ and $\lambda \Lambda \subseteq \gamma \Lambda$. Therefore $\Lambda^{-1}$ is a right valuation domain.

Certainly $\Lambda$ is a local ring without nonzero zero-divisors. By [2, Proposition 9.4] $\Lambda$ is a Noetherian ring. Let $I$ be a nonzero right ideal of $\Lambda$ and write $I=\sum_{i=1}^{t} \lambda_{i} \Lambda$. Then $I=\lambda_{i} \Lambda \cong \Lambda$ for some $1 \leqslant i \leqslant t$ since $\Lambda$ is a right valuation domain. Thus $\Lambda$ is a principal right ideal domain. Since $Q \Lambda$ is a division algebra $Q I=Q \Lambda$ and hence $\Lambda / I$ is finite. Let $k$ be the composition length of $\Lambda / I$ and let $J$ be the Jacobson radical of $\Lambda$. Since $\Lambda / J$ is the only simple $\Lambda$-module, $J^{k} \subset I$. Further, for positive integers $k, J^{k} \cong \Lambda$, so $J^{k} / J^{k+1} \cong \Lambda / J$. Thus $\Lambda \subset J \subset \ldots \subset J^{k}$ is a complete list of the right ideals of $\Lambda$ containing $J^{k}$. But then $I=J^{k}$ is an ideal of $\Lambda$, and so $\Lambda$ is a subcommutative ring. Finally, by Lemma $3.1 \mathrm{~b}$ and [2, Exercise 5.5, p. 57] $A$ is a finitely faithful E-flat group.

Reduced torsion-free finite rank valuation domains need not be commutative.

EXAMPLE 3.5. There is a noncommutative subcommutative right valuation domain $\Lambda$. 
Proof: Let $\Delta$ be the ring of Hamiltonian Quaternions over $Q$ and let $\Lambda$ be the unique classical maximal $\mathbb{Z}_{2}$-order in $\Delta$. Because 2 ramifies in $\Delta, \Lambda$ is a local ring whose Jacobson radical $J$ satisfies $J^{2}=2 \Lambda$. Further, each right ideal of $\Lambda$ is a power of $J$. (See $[\mathbf{1 7}$, Section 18$]$ for details.) Thus $\Lambda$ is a noncommutative subcommutative right valuation domain.

EXAMPLE 3.6. There is a finitely faithful E-flat torsion-free group $A$ of finite rank such that $\mathcal{L}(A)$ is a chain, but $\operatorname{End}(A)$ is not a commutative ring.

Proof: Use Corner's Theorem and Proposition 3.4 on the ring in Example 3.5.

We do not know of any universal conditions which force a subcommutative ring to be commutative.

\section{Hereditary RINGS}

In [5, Corollary 3.2] Arnold and Lady classify those torsion-free groups $A$ of finite rank for which $\Lambda$ is a right hereditary ring or a principal right ideal domain. Hereditary Noetherian endomorphism rings of torsion-free abelian groups have been studied extensively by Albrecht in a long series of papers culminating in [1]. Other studies include [12]. Theorem 1.2 provides us with intuitive classifications of those $A$ with (semi-)hereditary endomorphism rings.

The following extends [1, Proposition 2.2] and [12, Theorem 2.2]:

Theorem 4.1. Let $A$ be a group. Then the following are equivalent:

(a) $A$ is an $E$-flat group and $\Lambda$ is a right semi-hereditary ring;

(b) (i) $I A \neq A$ for finitely generated proper right ideals $I \subset \Lambda$; and (ii) finitely $A$-generated subgroups of $A$ are finitely $A$-projective groups;

(c) (i) $I A \neq A$ for finitely generated proper right ideals $I \subset \Lambda$; and (ii) finitely $A$-generated subgroups of finitely $A$-projective groups are finitely $A$-projective groups.

Proof: $(c) \Rightarrow(b)$ is clear.

(b) $\Rightarrow$ (a) Proceeding as in [1, Proposition 2.2], we first prove that $\Lambda$ is right semi-hereditary. Let $I$ be a finitely generated right ideal of $\Lambda$. By part (b) $I A$ is a summand of $A^{n}$ for some positive integer $n$ so we can write $A^{n}=I A \oplus B$ for some finitely $A$-generated group $B$. Identifying $I$ with a subset of $\mathcal{H}_{A}(I A) \subset \mathcal{H}_{A}\left(A^{n}\right)$ we have $\left(I \oplus \mathcal{H}_{A}(B)\right) A=A^{n}$. Because $\mathcal{H}_{A}(B)$ is a summand of $\mathcal{H}_{A}\left(A^{n}\right), I \oplus \mathcal{H}_{A}(B)$ is a finitely generated right $\Lambda$-submodule of $\mathcal{H}_{A}\left(A^{n}\right)$. By Lemma 2.3 and part (b.i), $I \oplus \mathcal{H}_{A}(B)=\mathcal{H}_{A}\left(A^{n}\right)$. Thus $I$ is a projective right $\Lambda$-module and $\Lambda$ is right semihereditary. 
To prove that $A$ is E-flat, recall that the map $\sigma: \mathcal{T}_{A}\left(\mathcal{H}_{A}\left(A^{n}\right)\right) \rightarrow A^{n}$ defined by $\sigma(\lambda \otimes a)=\lambda a$ is an isomorphism. Since $I \oplus \mathcal{H}_{A}(B)=\mathcal{H}_{A}\left(A^{n}\right)$ and since $\sigma\left(\mathcal{T}_{A}(I)\right)=$ $I A$, we have $\mathcal{T}_{A}(I) \cong I A$. That is, $A$ is E-flat.

(a) $\Rightarrow$ (c) Let $B \in \mathcal{L}\left(A^{n}\right)$ be finitely $A$-generated. Then there are maps $\lambda_{1}, \ldots, \lambda_{t} \in \mathcal{K}_{A}\left(A^{n}\right)$ such that $B=\sum_{i=1}^{t} \lambda_{i} A$. Let $H=\sum_{i=1}^{t} \lambda_{i} \Lambda$. Because $\Lambda$ is a right semi-hereditary ring there is a split embedding $\mathcal{S}: H \rightarrow \mathcal{H}_{A}\left(A^{t}\right)$. But then $B=H A \cong \mathcal{T}_{A}(H) \rightarrow \mathcal{T}_{A}\left(\mathcal{H}_{A}\left(A^{t}\right)\right) \cong A^{t}$ is a split embedding. This is (c.ii).

Next, assume $I$ is a finitely generated right ideal of $\Lambda$ such that $I A=A$. Then $I \in \sigma$ and so $\Lambda / I$ is $\sigma$-torsion. Because $\Lambda$ is a right semi-hereditary ring $I$ is a projective right $\Lambda$-module. Hence $I$ is $\sigma$-closed (Lemma 1.1c) and $\Lambda / I$ is $\sigma$-torsionfree. It follows that $\Lambda=I$. This proves (c.i) and completes the proof.

Corollaky 4.2. Let $A$ be a group. Then the following are equivalent:

(a) $A$ is an E-flat group and $\Lambda$ is a right hereditary, right Noetherian ring;

(b) $A$ is a finitely faithful group and each $B \in \mathcal{L}(A)$ is a finitely $A$-projective group.

Proof: Assume (a). Since each right ideal of $\Lambda$ is finitely generated Theorem 4.1b shows that $A$ is a finitely faithful group. Further, $\mathcal{L}(A)=\{I A \mid I$ is a right ideal of $\Lambda$ \} so that each $B \in \mathcal{L}(A)$ is finitely $A$-generated. Then by Theorem $4.1 \mathrm{~b}$ each $B \in \mathcal{L}(A)$ is a finitely $A$-projective group. This proves (b).

Assume (b). By Theorem 4.1 $A$ is a finitely faithful E-flat group and $\Lambda$ is a right semi-hereditary ring. Since a finitely $A$-projective group is a summand of a finitely $A$-free group, each $B \in \mathcal{L}(A)$ is finitely $A$-generated. Then by Corollary $3.2 \mathrm{a}, \Lambda$ is a right Noetherian ring, which proves part (a).

Call $A$ self-small if $\operatorname{Hom}\left(A, \oplus_{\Sigma} A\right) \cong \oplus_{\Sigma} \operatorname{Hom}(A, A)$ for each index set $\Sigma,[6]$. Torsion-free groups of finite rank are self-small. Self-small groups are used in [1] and [12] to classify groups with hereditary endomorphism rings.

THEOREM 4.3. Consider the following statements:

(a) $\Lambda$ is a right hereditary ring and $A$ is an $E$-flat group;

(b) $A$ is a finitely faithful group and each $B \in \mathcal{L}(A)$ is an $A$-projective group.

Then (a) $\Rightarrow$ (b). If $A$ is self-small then (b) $\Rightarrow$ (a).

Proof: Assume (a) and let $B \in \mathcal{L}(A)$. From Theorem 4.1, $A$ is a finitely faithful group, and by (a) there exists a right $\Lambda$-module $K$ and a set $\Sigma$ such that $\mathcal{H}_{A}(B) \oplus K^{-} \cong$ $\oplus_{\Sigma} \Lambda$. By Theorem 1.2 and since $\mathcal{T}_{A}(\cdot)$ commutes with direct sums, $B \cong \mathcal{T}_{A}\left(\mathcal{H}_{A}(B)\right)$ is a summand of $\oplus_{\Sigma} \mathcal{T}_{A}(\Lambda) \cong \oplus_{\Sigma} A$. This proves (b). 
Now assume $A$ is a self-small group satisfying (b). By Theorem 4.1, $A$ is an E-flat group. Let $I$ be a right ideal of $\Lambda$. By Corollary $2.2 \mathrm{~b}, I=\mathcal{H}_{A}(I A)$ and by (b) $I A$ is an $A$-projective group. Thus there is a group $C$ and an index set $\Sigma$ such that $I A \oplus C \cong \cong \oplus_{\Sigma} A$. Since $A$ is a self-small group, $I \oplus \mathcal{H}_{A}(C)=\mathcal{H}_{A}(I A) \oplus \mathcal{H}_{A}(C) \cong \oplus_{\Sigma} \Lambda$. Thus $\Lambda$ is a right hereditary ring. This proves (b) and completes the proof.

Remark. Using Proposition 2.4 it is seen that Theorems 4.1 and 4.3 classify the hereditary property in $\Lambda$ in terms of direct sum decomposition properties of $A$. Corollary 4.2 extends [1, Theorem 3.2] in that it removes the hypotheses that $A$ be a self-small, reduced torsion-free group. Corollary 4.2 and Theorem 4.3 show that a classification of groups with right hereditary endomorphism ring need not concern arbitary direct sums of copies of $A$. Also, these results demonstrate that [1, Theorem 3.2b.ii and iii] are not necessary to classify right hereditary, right Noetherian rings.

This Section ends with a classification of torsion-free groups of finite rank having heereditary endomorphism ring.

Conollary 4.4. Let $A$ be a reduced torsion-free group of finite rank. Then $\Lambda$ is a right hereditary ring if and only if $A$ is a finitely faithful group and each $B \in \mathcal{L}(A)$ is an $A$-projective group. In this case $A$ is an $E$-flat group, and $\Lambda$ is a hereditary Noetherian semi-prime ring.

Proof: By [2, Exercise 5.5, p. 57] $A$ is an E-flat group if $\Lambda$ is a right hereditary ring. Since torsion-free groups of finite rank are self-small groups, the equivalence follows from Theorem 4.3. The rest is [13, Theorem 2.3].

\section{MAXIMAL ORDERS AND NEAR ISOMORPHISM CLASSES}

Two torsion-free groups $A, B$ are nearly isomorphic if for each integer $n$ there are maps $f_{n}: A \rightarrow B$ and $g_{n}: B \rightarrow A$ and an integer $m=m(n)$ relatively prime to $n$ such that $f_{n} g_{n}=m 1_{B}$ and $g_{n} f_{n}=m 1_{A}$. See [2] or [14] for properties of near isomorphism. We will call $A$ integrally closed if $A=\operatorname{Hom}(B, A) B$ for each $B \in \mathcal{L}(A)$ of finite index in $A$.

Our goal is to classify, in terms of $\Lambda$, those torsion-free groups $A$ of finite rank which are nearly isomorphic to each $B \in \mathcal{L}(A)$ of finite index in $A$. The lemmas will prove useful.

LEMMA 5.1. Assume $A$ is a torsion-free group of finite rank.

(a) If $\mathrm{Q} \Lambda$ is a semi-simple ring, then each $B \in \mathcal{L}(A)$ is a quasi-summand of A.

(b) $Q \Lambda$ is a simple ring if and only if $\operatorname{Hom}(B, A) B$ has finite index in $A$ for each $B \in \mathcal{L}(A)$. 
Proof: (a) Let $B \in \mathcal{L}(A)$. Then $Q \mathcal{H}_{A}(B)=\mathbb{Q H o m}(A, B) \neq 0$. Because $\mathrm{Q} \Lambda$ is semi-simple, $\mathrm{QH}_{A}(B)$ is a summand of $\mathrm{Q} \Lambda$, so that $e \mathrm{Q} \Lambda=\mathrm{QH}_{A}(B)$ for some idempotent $e$ of $Q \Lambda$. Then $n e \in \mathcal{H}_{A}(B)$ for some positive integer $n$ and $\mathcal{H}_{A}(B) \cap$ $n(1-e) \Lambda=0$, so $n \Lambda \subseteq \mathcal{H}_{A}(B) \oplus n(1-e) \Lambda \subseteq \Lambda$. But then $B \cap n(1-e) A=0$ and $n A \subseteq B \oplus n(1-c) A \subseteq A$, so that $B$ is a quasi-summand of $A$.

(b) Assume that $\operatorname{Hom}(B, A) B$ has finite index in $A$ for each $B \in \mathcal{L}(A)$, and let $I$ be a nonzero ideal of $\Lambda$. An application of $\operatorname{Hom}(\cdot, A)$ to the multiplication map $\mathcal{T}_{A}(I) \rightarrow I A \rightarrow 0$ yields an embedding

$$
\operatorname{Hom}(I A, A) \rightarrow \operatorname{Hom}\left(\mathcal{T}_{A}(I), A\right) \cong \operatorname{Hom}_{\Lambda}(I, \Lambda)
$$

of left $\Lambda$-modules, where the isomorphism is adjointness. Let $J$ be a left ideal of $\Lambda$ such that $J I=0$. Then $J f(I)=f(J I)=0$ for each $f \in \operatorname{Hom}_{\Lambda}(I, \Lambda)$, and hence $J \operatorname{Hom}(I A, A)=0$. Now $A / \operatorname{Hom}(I A, A) I A$ is finite by hypothesis and $A$ is a faithful left $\Lambda$-module so $J A=0=J$. That is, each nonzero ideal of $\Lambda$ has zero left annihilator. Since the Jacobson radical of $Q \Lambda$ is nilpotent, $Q \Lambda$ must be semi-simple. Finally, the Wedderburn Theorem easily shows that $Q \Lambda$ is simple.

Conversely, if $\mathrm{Q} \Lambda$ is a simple ring, then given $B \in \mathcal{L}(A), \operatorname{Hom}(B, A) B=C$ is a fully invariant subgroup of $A$. Hence, $Q \mathcal{H}_{A}(C)$ is an ideal of $\mathbf{Q} \Lambda$. By hypothesis, $\mathrm{Q} \Lambda=\mathrm{QH}_{A}(C)$ so that $\mathcal{H}_{A}(C)$ contains a nonzero integer $n$. Thus $n A \subseteq C \subseteq A$, which completes the proof.

Let $M, N$ be right $\Lambda$-modules which are torsion-free groups. Then $M$ is in the genus class of $N$ if for each integer $n \neq 0$ there are $\Lambda$-module homomorphisms $f_{n}: M \rightarrow N$ and $g_{n}: N \rightarrow M$ and an integer $m=m(n)$ which is relatively prime to $n$ such that $f_{n} g_{n}=m 1_{N}$ and $g_{n} f_{n}=m 1_{M}$. In [4] and [2, Chapter 12] Arnold develops the connection between near isomorphism classes of $A$-projective groups and the genus classes of projective right $\Lambda$-modules. Specifically, [2, Corollary 12.7] shows that $\mathcal{H}_{A}(\cdot)$ induces a one-to-one correspondence between near isomorphism classes of finitely $A$-projective groups and the genus classes of finitely generated projective right $\Lambda$-modules. We extend [2, Corollary 12.7] to the larger classes $\mathcal{S}(A)$ and $\mathcal{F}(\Lambda)$.

LeMma 5.2. Let $A$ be a torsion-free E-flat group. Let $B, C \in \mathcal{S}(A)$. Then $B$ is isomorphic to (respectively, nearly isomorphic to) $C$ if and only if $\mathcal{H}_{A}(B)$ is isomorphic to (respectively, in the genus class of) $\mathcal{H}_{A}(C)$.

PROOF: By Theorem 1.2a, there are canonical isomorphisms

$$
\operatorname{Hom}(B, C) \cong \operatorname{Hom}_{\Lambda}\left(\mathcal{H}_{A}(B), \mathcal{H}_{A}(C)\right)
$$

and

$$
\operatorname{Hom}(C, B) \cong \operatorname{Hom}_{\Lambda}\left(\mathcal{H}_{A}(C), \mathcal{H}_{A}(B)\right)
$$


Thus $B$ is isomorphic to (respectively, nearly isomorphic to) $C$ if and only if $\mathcal{H}_{A}(B)$ is isomorphic to (respectively, in the genus class of) $\mathcal{H}_{A}(C)$.

We will call $\Lambda$ an integrally closed ring if (i) $\Lambda$ is a torsion-free finite rank semiprime ring and if (ii) $\Lambda=\Gamma$ whenever $\Gamma$ is a subring of $Q \Lambda$ that contains $\Lambda$ as a subring of finite index. A classical maximal order is an integrally closed prime ring. See [2, Chapters 9-12] for properties of integrally closed rings, and [17] for properties of classical maximal orders. Each integrally closed ring is a finite product of classical maximal orders, and each torsion-free semi-prime ring is of finite index in an integrally closed ring [2, Corollary 10.14c]. Classical maximal orders and hence integrally closed rings are hereditary Noetherian rings, [2, Theorem 11.3].

Proposition 5.3. Let $\Lambda$ be a reduced torsion-free finite rank ring. Then $\Lambda$ is an integrally closed ring if and only if each right ideal of finite index in $\Lambda$ is in the genus class of $\Lambda$.

Proof: Assume $\Lambda$ is integrally closed and let $I$ be a right ideal of finite index in $\Lambda$. Then $\Lambda=\Lambda_{1} \times \ldots \times \Lambda_{t}$ for some classical maximal orders $\Lambda_{1}, \ldots, \Lambda_{t}$, and $I=I_{1} \times \ldots \times I_{t}$ where, for each $i, I_{i}$ is a right ideal of finite index in $\Lambda_{i}$. By [2, Theorem 11.8] $\left(\Lambda_{i}\right)_{p}$ is a principal right ideal ring for each prime $p \in \mathbb{Z}$. Hence $\left(I_{i}\right)_{p}=\lambda\left(\Lambda_{i}\right)_{p}$ for some $\lambda \in \Lambda_{i}$. Because $\Lambda_{i} / I_{i}$ is finite and because $\Lambda_{i}$ has finite rank, $\left(\Lambda_{i}\right)_{p} \cong\left(I_{i}\right)_{p}$. Then $\left[2\right.$, Theorem 12.2] shows that $I_{i}$ is in the genus class of $\Lambda_{i}$ and hence that $I$ is in the genus class of $\Lambda$.

Conversely, assume each right ideal of finite index in $\Lambda$ is in the genus class of $\Lambda$, and let $I$ have finite index in $\Lambda$. Then $n \Lambda \subseteq I$ for some nonzero integer $n$. Since $\Lambda / I$ is finite, $I$ is a finitely generated right $\Lambda$-module. It follows easily from the definition of genus class that for each prime $p \in Z$ there are isomorphisms $I_{p} \cong \Lambda_{p}$. By the Local-global Theorem, $I$ is a projective right $\Lambda$-module. Then using the proof of $[13$, Theorem 2.3] it is sliown that $\Lambda$ is a semi-prime ring. Hence, there is an integrally closed subring $\Lambda \subset \Gamma \subset \mathrm{Q} \Lambda$ such that $\Gamma / \Lambda$ is finite, [2, Corollary 10.14c]. By hypothesis $\Gamma$ is in the genus class of $\Lambda$, so for each prime $p \in \mathbb{Z}$, there exists a $\gamma \in \Gamma_{p}$ such that $\Gamma_{p}=\gamma \Lambda_{p} \cong \Lambda_{p}$. Evidently $\gamma$ is a unit of $Q \Lambda$, so $\Gamma_{p}=\left\{\lambda \in Q \Lambda \mid \lambda \Gamma_{p} \subset \Gamma_{p}\right\}=$ $\gamma \Lambda_{p} \gamma^{-1} \cong \Lambda_{p}$. Since $\Lambda_{p}$ is then integrally closed, [2, Theorem 11.7], $\Gamma_{p}=\Lambda_{p}$ for each prime $p \in \mathbb{Z}$. By the Local-global Theorem $\Gamma=\Lambda$, and $\Lambda$ is integrally closed.

THEOREM 5.4. Let $A$ be a reduced torsion-free group of finite rank. Then the following are equivalent:

(a) $\Lambda$ is an integrally closed ring;

(b) $A$ is a finitely faithful E-flat group and $A$ is nearly isomorphic to each $B \in \mathcal{L}(A)$ of finite index in $A$;

(c) $\Lambda$ is a semi-prime ring and $A$ is an integrally closed group. 
Proof: (a) $\Rightarrow$ (b) Since $A$ is a torsion-free group of finite rank, and since $\Lambda$ is a hereditary ring, Corollary 4.3 shows $A$ is a finitely faithful E-flat group. By Proposition 5.3 each right icleal of finite index in $\Lambda$ is in the genus class of $\Lambda$, and by Proposition 2.1 each right ideal $I$ of $\Lambda$ satisfies $I=\mathcal{H}_{A}(I A)$. Since $\mathcal{L}(A)=\{I A \mid I \in \mathcal{L}(\Lambda)\}$, Lemma 5.2 shows that $A$ is nearly isomorphic to each $B \in \mathcal{L}(A)$ of finite index in $A$.

(b) $\Rightarrow$ (c) Given a right ideal $I$ of finite index in $\Lambda$, Proposition 2.1 shows that $I$ is a homset. As $A / I A$ is then finite, part (b) states that $A$ is nearly isomorphic to $I A$. By Lemma 5.2, $I=\mathcal{H}_{A}(I A)$ is in the genus class of $\Lambda=\mathcal{H}_{A}(A)$, so by Proposition 5.3 $\Lambda$ is a semi-prime ring. Next let $B \in \mathcal{L}(A)$ have finite index in $A$. By hypothesis $B$ is nearly isomorphic to $A$, so there is an integer $k>0$ such that $B^{k} \cong A^{k},[2$, Theorem 13.9]. Thus $A$ is integrally closed.

(c) $\Rightarrow$ (a) Since $\Lambda$ is a semi-prime ring there is an integrally closed subring $\Gamma$ of $Q \Lambda$ containing $\Lambda$ such that $\Gamma / \Lambda$ is finite, [2, Corollary 10.14c]. Consider $A \subseteq \Gamma A \subseteq Q A$ and note that $\Gamma A / A$ is finite. Then by part (c) $\mathcal{H}_{\Gamma A}(A) \Gamma A=A \in \mathcal{L}(\Gamma A)$. Now $\Lambda \subseteq \Gamma \subseteq \operatorname{End}(\Gamma A)$ and $\Gamma A / A$ is finite so $\operatorname{End}(\Gamma A) / \Lambda$ is finite. Hence End $(\Gamma A) / \Gamma$ is finite, and because $\Gamma$ is integrally closed $\Gamma=\operatorname{End}(\Gamma A)$. As $\Gamma$ is a hereditary ring, $\Gamma A$ is a finitely faithful E-flat group, (Corollary 4.4). Now $\Gamma$ is a product of classical maximal orders and $\mathcal{H}_{\Gamma A}(A)$ has finite index in $\Gamma$, so it readily follows from $[\mathbf{1 7}$, Exercise 1, p. 90] that

$$
\operatorname{End}_{\Gamma}\left(\mathcal{H}_{\Gamma A}(A)\right)=\left\{\gamma \in Q \Gamma \mid \lambda \mathcal{H}_{\Gamma A}(A) \subseteq \mathcal{K}_{\Gamma A}(A)\right\}
$$

is an integrally closed ring. Finally, since $\mathcal{H}_{\Gamma A}(\cdot)$ is an equivalence, there is an isomorphism of rings $\operatorname{End}(A) \cong \operatorname{End}_{\Gamma}\left(\mathcal{H}_{\Gamma A}(A)\right)$, (Theorem 1.2a). This proves (a) and completes the proof.

COROLLARY 5.5. Let $A$ be a reduced torsion-free group of finite rank, and assume $\Lambda$ is a semi-prime ring. Then the following are equivalent:

(a) $\Lambda$ is an integrally closed ring;

(b) $A$ is nearly isomorphic to each $B \in \mathcal{L}(A)$ of finite index in $A$;

(c) $A$ is an integrally closed group.

Proof: (a) $\Leftrightarrow($ c) $\Rightarrow$ (b) is Theorem 5.4. To prove (b) $\Rightarrow$ (c) use the proof of $5.4(\mathrm{~b}) \Rightarrow$ (c) .

There is an especially neat classification of maximal orders along these lines.

Corollary 5.6. Let $A$ be a reduced torsion-free group of finite rank. Then the following are equivalent:

(a) $\Lambda$ is a classical maxinal order;

(b) $A$ is generated by each nonzero $B \in \mathcal{L}(A)$; 
(c) $A$ is an integrally closed group and $A / \operatorname{Hom}(B, A) B$ is finite for each nonzero $B \in \mathcal{L}(A)$.

Proof: (a) $\Leftrightarrow$ (c) is Theorem 5.4 and Lemma 5.1b while (b) $\Leftrightarrow$ (c) is Lemma $5.1 \mathrm{~b}$.

\section{PRINCIPAL RIGHT IDEALS AND ISOMORPHISM CLASSES}

We specialise Theorem 5.4 to principal right ideal rings.

Proposition 6.1. Let $A$ be a finitely faithful E-flat group. Let $H \subset$ $\operatorname{Hom}\left(A, A^{n}\right)$ and let $F$ be a subset of $H$. Then $F \Lambda=H$ if and only if $F A=H A$. Specifically, $H$ is generated by $n$ elements as a $\Lambda$-module if and only if $H A$ is the sum of $n$ homomorphic images of $A$.

Proof: By Corollary 2.2b, $F \Lambda=H$ if and only if $F A=(F \Lambda) A=H A$. The rest is then clear.

Theorem 6.2. Let $A$ be a reduced torsion-free group of finite rank. Then the following are equivalent:

(a) $\Lambda$ is a principal right ideal ring;

(b) $A$ is a finitely faithful E-flat group and $A$ is isomorphic to each $B \in \mathcal{L}(A)$ of finite index in $A$;

(c) $\Lambda$ is a semi-prime ring and each $B \in \mathcal{L}(A)$ is an endomorphic image of A.

Proof: (a) $\Rightarrow$ (b) Let $I$ be a right ideal of finite index in $\Lambda$. Then $I=\lambda \Lambda$ for some $\lambda \in \Lambda$. Since $\Lambda$ has finite rank, $\lambda: \Lambda \rightarrow I$ is an isomorphism of right $\Lambda$-modules. By Proposition 5.3 $\Lambda$ is an integrally closed ring, so by Theorem $5.4 A$ is a finitely faithful E-flat group. Lemma 5.2 shows that $A$ is isomorphic to each $B \in \mathcal{L}(A)$ of finite index in $A$.

(b) $\Rightarrow$ (c) By Theorem $5.4 \Lambda$ is an (integrally closed) semi-prime ring. Given $B \in \mathcal{L}(A)$, Lemma 5.1 a produces a $C \in \mathcal{L}(A)$ such that $B \oplus C$ has finite index in $A$. By part (b) $A \cong B \oplus C$, so $B$ is an endomorphic image of $A$.

(c) $\Rightarrow$ (a) As in Theorem 5.4 (c) $\Rightarrow$ (a), there is an integrally closed ring $\Lambda \subset$ $\Gamma \subset \mathrm{Q} \Lambda$ such that $\Gamma / \Lambda$ is finite and $\operatorname{End}(\Gamma A)=\Gamma$. By hypothesis $\Gamma A=\lambda A$ for some $\lambda \in \operatorname{Hom}(A, \Gamma A)$, and because $A$ has finite rank, $\lambda: A \rightarrow \Gamma A$ is an isomorphism. Hence $A \cong \Gamma A$ and $\Lambda \cong \Gamma$. By Theorem 5.4, $A$ is a finitely faithful E-flat group, so by (c) and Proposition 6.1, each right ideal of $\Lambda$ is principal. This completes the proof. 
COROLlary 6.3. Let $A$ be a reduced torsion-free group of finite rank and assume $\Lambda$ is a semi-prime ring. Then the following are equivalent:

(a) $\Lambda$ is a principal right ideal ring;

(b) $A$ is isomorphic to each $B \in \mathcal{L}(A)$ of finite index in $A$;

(c) Each $B \in \mathcal{L}(A)$ is an endomorphic image of $A$.

Proof: (a) $\Leftrightarrow$ (c) $\Rightarrow$ (b) is Theorem 6.2. To prove (b) $\Rightarrow$ (c) argue as in Theorem $6.2(b) \Rightarrow(c)$.

Remark. The results of Theorems 5.4,6.2 and related Corollaries are partial solutions to the problem of deciding when quasi-isomorphic torsion-free groups are (nearly) isomorphic. Call $A$ an $S$-group if $\mathcal{L}(A)$ contains each subgroup of finite index in $A$, and call $A$ a $J$-group if $A$ is isomorphic to each subgroup of finite index in $A$. The following is a composite of results from [3] and [9]:

COMPOSITE RESULTS 6.4. The following are equivalent for torsion-free groups of finite rank:

(a) $A$ is a finitely faithful $S$-group;

(b) $p-\operatorname{rank}(A)^{2}=p-\operatorname{rank}(\Lambda)$ for all primes $p \in \mathbb{Z}$, where $p-\operatorname{rank}(G)$ is the $\mathbb{Z} / \mathbb{Z} p$-dimension of $G / G p$

(c) $\Lambda$ is semi-prime and $A$ is nearly isomorphic to each subgroup of finite index in $A$.

COMPOSITE RESUlTs 6.5. If $p-\operatorname{rank}(A) \neq 2$ for each prime $p$, then the following are equivalent:

( $\alpha) \quad A$ is a finitely faithful $S$-group;

( $\beta$ ) $\Lambda$ is a semi-prime ring and $A$ is a $J$-group.

It is clear from properties (b), (c) and $(\beta)$ that the finitely faithful S-group property and the $J$-group property are consequences of an interplay of the group structure of $A$ and the ideal structure of $\Lambda$. This is in marked contrast to Theorems 5.4 and 6.2 which show that weaker versions of the J-group and S-group property can be classified in terms of the ideal structure of $\Lambda$ alone.

\section{REFERENCES}

[1] U. Albrecht, 'Baer's lemma and Fuch's problem 84a', Trans. Amer. Math. Soc. 283 (1986), 565-582.

[2] D.M. Arnold, Finite Rank and Torsion-free Abelian Groups and Rings, Lecture Notes in Mathematics 931 (Springer-Verlag, New York, 1982).

[3] D.M. Arnold, 'Endomorphism rings and subgroups of finite rank torsion-free abelian groups', Rocky Mountain J. Math. 12 (1982), 241-256. 
[4] D.M. Arnold, Genera and Direct Sum Decompositions of Torsion-free Modules, Lecture Notes in Mathematics 616, pp. 197-218 (Springer-Verlag, New York, 1976).

[5] D.M. Arnold and E.L. Lady, 'Endomorphism rings and direct sums of torsion-free abelian groups', Trans. Amer. Math. Soc. 211 (1975), 225-237.

[6] D.M. Arnold and C.E. Murley, 'Abelian groups $A$, such that $\operatorname{Hom}(A,-)$ preserves $\operatorname{direct} \operatorname{sums}$ of copies of A', Pacific J. Math. 56 (1975), 7-20.

[7] V. Camillo and K.R. Fuller, 'Rings whose faithful modules are flat', Archiv. Math (Basel) 27 (1976), 522-525.

[8] T.G. Faticoni and P. Goeters, 'Examples of torsion-free abelian groups flat as modules over thier endomorplism ring', Proc. London Math. Soc. (to appear).

[9] T.G. Faticoni and P. Goeters, 'On torsion-free Ext', Comm. Alg. (1988) (to appear).

[10] T.G. Faticoni, 'Semi-local localizations of rings and subdirect decompositions of modules', J. Pure Appl. Alg. 46 (1987), 137-163.

[11] L. Fuchs, Infinite Abelian Groups,: Volumes $I$ and $I I$ (Academic Press, New York London, 1970, 1973).

[12] J. Hausen, 'Modules with the summand intersection property', (preprint).

[13] M. Huber and R.B. Warfield, Jr., Homomorphisms between Cartesian Powers of an Abelian Group, Lecture Notes in Mathematics 874, pp. 202-227 (Springer-Verlag, New York, 1981).

[14] L. Lady, 'Nearly isomorphic torsion-free abelian groups', $J$. Algebra 35 (1975), 235-238.

[15] J. Reid, 'On subcommutative rings', 'Acta. Math. Acad. Sci. Hungar. 16 (1965), 23-26.

[16] J. Reid, 'On the ring of quasi-endomorphisms of a torsion-free group', in Topics in Abelian Groups, pp. 51-68 (Chicago, Illinois, 1963).

[17] I. Reiner, Maximal Orders (Academic Press, New York, 1975).

[18] F. Richman and E.A. Walker, 'Primary abelian groups as modules over their endomorphisın rings', Math $Z 88$ ((1965)), 77-81.

[19] B. Stenström, Rings of Quotients (Springer-Verlag, Berlin, Heidelberg, New York, 1975).

Department of Mathematics,

Fordham University,

Bronx NY. 10458,

United States of America. 